\title{
]jfis
}

\section{Hypergroupoids as Tools for Studying Blood Group Genetics}

\author{
Mohammad Munir ${ }^{1}$, Nasreen Kausar ${ }^{2}$, Salahuddin ${ }^{3}$, Rukhshanda Anjum ${ }^{4}$, \\ Qingbing $\mathrm{Xu}^{5}$, and Waqas Ahmad ${ }^{6}$ \\ ${ }^{1}$ Department of Mathematics, Government Postgraduate College, Abbottabad, Pakistan \\ ${ }^{2}$ Department of Mathematics, Faculty of Arts and Science, Yildiz Technical University, Istanbul, Turkey \\ ${ }^{3}$ Department of Mathematics, Jazan University, Jazan, Kingdom of Saudi Arabia \\ ${ }^{4}$ Department of Mathematics and Statistics, University of Lahore, Lahore \\ ${ }^{5}$ Department of Basic Courses, Chuzhou Polytechnic College, Chuzhou, China \\ ${ }^{6}$ Department of Zoology, Hazara University, Mansehra, Pakistan
}

Received: Jan. 30, 2021

Revised : May 3, 2021

Accepted: May 28, 2021

Correspondence to: Nasreen Kausar (kausar.nasreen57@gmail.com)

(T) The Korean Institute of Intelligent Systems

CCThis is an Open Access article distributed under the terms of the Creative Commons Attribution Non-Commercial License http://creativecommons.org/licenses/by-nc / 3.0/) which permits unrestricted noncommercial use, distribution, and reproduction in any medium, provided the original work is properly cited.

\begin{abstract}
We initially introduce the concepts of an $m$-right ( $m$-left) hyperideal and an $m$-hyperideal in a hypergroupoid. The ideas behind an $m$-factor and a generalized $m$-factor are then introduced. Next, we demonstrate the existence and important properties of these sub-hyperstructures through theorems and examples. We then define the $m$-right ( $m$-left) consistent, $m$-consistent, $m$-intra-consistent, and $m$-simple hypergroupoids. Finally, we demonstrate that practical problems in biology, such as $A B O$ blood group genetics, can be studied by defining these hypergroupoid substructures.
\end{abstract}

Keywords: Blood group genetics, $m$-factors, Genotype, Phenotype, $m$-factorizable, Hypersemigroups

\section{Introduction}

Among all algebraic structures, a groupoid is the simplest within the entire field of mathematics, consisting of a non-empty set, $H$, with a single binary operation, $\cdot$, defined based on its elements, which are usually denoted by $(H, \cdot)$, for which the associativity condition does not generally hold. For this reason, groupoids cover a large number of applications in mathematics. Such applications are suitable for studying systems that do not necessarily satisfy the associative laws under a binary operation. In terms of a binary operation is considered, algebraic systems that assign more than one value to an ordered pair under a function have also not been studied. The study of blood groups is one such example that does not come under the fold of associative systems with binary operations. However, these problems that do not obey the associative laws and do not admit binary operations are of considerable importance in our daily life. Through their subsets and ideals, particularly $m$ ideals, we can study the physical systems behind them in a more understandable way. The groupoids naturally lead us to study hyperstructures called hypergroupoids, which are a natural generalization of groupoids [1]. Moreover, the most appropriate hyperstructure for the study of blood groups is a study of hypergroupoids. In addition, to study the influence of blood groups from a population of parents to a population of offspring, i.e., the so-called blood group genetics, the $m$-hyperideals provide more information than the hyperideals, where $m$ may be termed the generation number. 
This issue motivated us to pursue the study of $m$-hyperideals in hypergroupoids in association with the study of blood groups.

Marty [2] introduced hyperstructures in 1934. Later mathematicians then followed up on this study for different algebraic structures and applied them to study various problems in scientific fields. For example, Hasankhani [3] studied the ideals with respect to Greens's relation in hypersemigroupoids. Kehayopulu [4] characterized hypergroupoids through the properties of fuzzy prime and fuzzy semiprime hyperideals. Suebsung et al. [5] characterized the semihypergroupoids through the properties of almost hyperideals.

The motivation to work on the so-called $m$-hyperideals is based on the description above. In essence, having come across examples that do not obey the associative laws under the given binary operation, and owing to a lack of binary operations and studies on algebraic systems that assign more than one element to an ordered pair, there is a need to pursue studies on hypergroupoids with special reference to generalizing their hyperideals. Regarding the pattern in generalizing the ideals during our research on the semigroup theory [67] and the semiring theory [8], and the fuzzy ideals [6], in this article, we generalize the hyperideals of hypergroupoids through a positive integer $m$, and then discuss the concepts arising from the consequences based on examples and their applications.

This paper is divided into seven sections. In Section 1, we provide the necessary introduction and motivation of our study. Section 2 presents the concepts necessary to be used in further research. In Section 3 , we introduce the idea of $m$-hyperideals in hypergroupoids along with their different properties and examples. Alongside the $m$-hyperideals, in Section 4, we present the idea of $m$-factorizable hypergroupoids and associated concepts. Section 5 deals with the genetics of ABO blood groups, as described by the method of hypergroupoids. Section 6 deals with the $m$-simple hypergroupoids, and Section 7 presents some concluding remarks regarding our study.

\section{Preliminaries}

In this section, we present some preliminary ideas from the literature on hypergroupoids, which will be necessary to build up the theory and applications of $m$-hyperideals. A non-empty set $H$, along with a binary operation - defined on its elements, is called a groupoid and is denoted by $(H, \cdot)$. For a groupoid $H$, we denote by $P^{*}(H)$ the set of all non-empty subsets of $H$. Then, $P^{*}(H)$ becomes a groupoid with the binary operation defined on its elements by $A B=\{a b ; a \in A, b \in B\}$ for all $A, B \in P^{*}(H)$ [9]. A non-empty subset $S$ of $H$ such that $a, b \in S$ for all $a, b \in S$ is called a subgroup of $H$. For a positive integer $m$, we can generate different subgroupoids of $H$ as particular elements of $P^{*}(H)$ from $H$ by taking the different powers denoted by $H^{m}$, as defined below.

Definition 2.1. Following [10] and [5], let $H$ be a non-empty set, and the map $\circ: H \times H \rightarrow P^{*}(H)$ is then called a hyperoperation, where $P^{*}(H)$ is the family of all non-empty subsets of $H$.

Definition 2.2. We define a hypergroupoid as an ordered pair $(H, \circ)$ consisting of a non-empty set $H$ and a hyperoperation $\circ: H \times H \rightarrow P^{*}(H)$, where $P^{*}(H)$ is the set of all non-empty subsets of $H$, defined by

$$
S \circ K=\bigcup_{a \in S, b \in K}(a \circ b),
$$

for all non-empty subsets $S$ and $K$ of $H$.

We denote the hypergroupoid $(H, \circ)$ simply by $H$. It follows from the definition that $a \circ K=\{a\} \circ H$ and $H \circ a=H \circ\{a\}$. In addition, $e$ is called a left identity if $a \circ e=e$, and $e$ is called the right identity if $e \circ a=\{a\}$ for all $a \in H$. Moreover, $e$ is called a scalar identity or simply an identity if $a \circ e=e \circ a=\{a\}$ for all $a \in H$. The identity of $H$ is unique by definition. An element $a \in H$ is idempotent if $a \circ a=a$. Here, $H$ is said to be commutative if $a \circ b=b \circ a$ for all $a, b \in H$. A hypergroupoid $(H, \circ)$ is said to be a hypersemigroup if the hyperoperation $\circ$ follows the associative law on all elements of $H$, that is, if $a \circ(b \circ c)=(a \circ b) \circ c$ for every $a, b, c \in H$.

A non-empty subset $S$ of $H$ is known as its subhypergroupoid if $a, b \in S$ implies $a \circ b \subseteq S$, and comparably, $S \circ S \subseteq S$ implies that $S$ is a subhypergroupoid of $H$. A non-empty subset $S$ of a hypergroupoid $H$ is said to be a right (left) hyperideal of $H$ if $S \circ H \subseteq S$ ( $H \circ S \subseteq S$ ). A non-empty set, $T$, is called a two-sided hyperideal, or simply a hyperideal of $H$, if it is both a left hyperideal and a right hyperideal of $H$, that is, $H \circ T \circ H \subseteq T$.

For a positive integer $m, H^{m}=\underbrace{H \circ H \circ H \circ \cdots \circ H}_{m \text {-times }}$. Moreover, $H^{2}=H \circ H \subseteq H$ (defined by Definition 2.2. Continuing in this manner, we have $H^{l} \subseteq H^{n}$ for $l, m$ as two positive integers such that $l \geq m$. In addition, $H^{m}$ is a subhypergroupoid of $H$ for all values of $m$, and $H^{m+n}=H^{n} \circ H^{m}$ for any two positive integers $m$ and $n$.

The condition $S \circ H \subseteq S(H \circ S \subseteq S$ ) is equivalent to $s \circ h \subseteq S(h \circ s \subseteq S$ ) for every $s \in S$ and $h \in H$. 
Definition 2.3. A hypergroupoid $H$ is said to be left (right) simple if and only if $H$ does not possess any proper left (right) hyperideal. In addition, $H$ is said to be simple if it does not have a hyperideal.

Definition 2.4. A hypergroupoid $H$ is termed as right-consistent if $(a \circ b) \circ H=a \circ(b \circ H)$ for all $a, b \in H$. Here, $H$ is called left consistent if $H \circ(a \circ b)=(H \circ a) \circ b$ for all $a, b \in H$. If $H$ is both left and right consistent, then it is called a consistent hypergroupoid [11].

Definition 2.5. A hypergroupoid $H$ is said to be inconsistent if $(a \circ H) \circ b=a \circ(H \circ b)$ for all $a, b \in H$.

Definition 2.6. An element $a$ of a hypergroupoid $H$ is said to be a weak right (left) magnifying element of $H$ if there exists a proper subset $K$ of $H$ such that $H=K \circ a(H=a \circ K)$. Moreover, $a$ is said to be a strong right (left) magnifying element of $H$ if there exists a proper subhypergroupoid $S$ of $H$ such that $H=S \circ a(H=a \circ S)[12]$.

It should be noted that the identity and regular elements of a hypergroupoid are not weak right or weak left magnifying elements.

Definition 2.7. We define the principal left hyperideal as the left hyperideal $H \circ a=\langle a\rangle_{l}$ generated by $a \in H$, and the principal right ideal as $a \circ H=\langle a\rangle_{r}$. The principal two-sided ideal generated by $a$ is characterized as $H \circ a \circ H=\langle a\rangle$.

\section{3. m-Hyperideals in Hypergroupoid}

Definition 3.1. A non-empty subset $L(R)$ of a hypergroupoid $(H, \circ)$ is called an $m$-left hyperideal ( $m$-right hyperideal) of $H$ if $H^{m} \circ L \subseteq L\left(R \circ H^{m} \subseteq R\right)$, where $m$ is a positive number. Equivalently, if for each $a \in L(a \in R)$ and each $h \in H^{m}$, we have $a \circ h \subseteq L(h \circ a \subseteq R)$.

A non-empty set $T$ of $H$ is called an $m$-two-sided hyperideal or simply $m$-hyperideal of $H$ in the event that it is both an $m$ left hyperideal and an $m$-right hyperideal of $H$, that is, $H^{m} \circ$ $T \circ H^{m} \subseteq T$.

Remark 3.2. Every left hyperideal is an $m$-left hyperideal of $H$ (for $m=1$ ), but the converse does not follow. Similar statements hold for the $m$-right hyperideals and $m$-hyperideals of $H$. This is evident in Example 3.4

Definition 3.3. The principal $m$-left hyperideal generated by an element $a \in H$ is the $m$-left hyperideal $H^{m} \circ a$. The principal $m$-right ideal generated by $a$ is characterized as $a \circ H^{m}$. The principal two-sided $m$-ideal generated by $a$ is characterized as $H^{m} \circ a \circ H^{m}$.
Table 1.

\begin{tabular}{cccc}
\hline$\circ$ & 1 & 2 & 3 \\
\hline 1 & $\{1\}$ & $\{1,2\}$ & $\{2\}$ \\
2 & $\{1\}$ & $\{2\}$ & $\{1\}$ \\
3 & $\{1,2\}$ & $\{2\}$ & $\{1\}$ \\
\hline
\end{tabular}

Example 3.4. Consider the hypergroupoid $H=\{1,2,3\}$ with the hyperoperation $\circ$ defined in Table 1 . Then, $H^{2}=$ $\{1,2\}$. The subhypergroupoid $\{1\}$ is a 2 -left hypergroupoid as $H^{2} \circ\{1\}=\{1\} \subseteq\{1\}$, whereas $\{1\}$ is not a left hyperideal of $H$ as $H \circ\{1\}=\{1,2\} \not\{\{1\}$.

Theorem 3.1. The product of an $m$-left $(m$-right) hyperideal and an $n$-left( $n$-right) hyperideal of $H$ is a $\max (m, n)$-left(max $(m, n)$-right) hyperideal of $H$, and $m$ and $n$ are any two positive integers.

Proof. Let $L_{1}$ be $m$-left and $L_{2}$ be $n$-left hyperideals of $H$. Consider

$$
\begin{aligned}
H^{\max (m, n)} \circ L_{1} \circ L_{2} \subseteq & H^{m} \circ L_{1} \circ L_{2} \subseteq L_{1} \circ L_{2}, \\
& \left(\text { since } H^{\max (m, n)} \subseteq H^{m}\right) .
\end{aligned}
$$

Thus, $L_{1} \circ L_{2}$ is $\max (m, n)$-left hyperideal of $H$. Similarly, the result follows for the $m$-left and $n$-right hyperideals.

Corollary 3.5. The product of two $m$-left ( $m$-right) hyperideals of $H$ is an $m$-left ( $m$-right) hyperideal of $H$.

Proof. Straightforward.

Theorem 3.2. The product of an $m$-hyperideal and $n$-hyperideal of $H$ is an $\max (m, n)$-hyperideal of $H$.

Proof. Because the product is both an $m$-left hyperideal and an $m$-right hyperideal, this product is $\max (m, n)$-hyperideal.

Corollary 3.6. The product of the $m$-two-sided hyperideals of hypergroupoid $H$ is an $m$-two-sided hyperideal of $H$.

Proof. Straightforward.

Remark 3.7. Any finite collection of $m$-left $(m$-right, $m$ two-sided) hyperideals of a hypergroupoid $H$, taken in any order, is also an $m$-left ( $m$-right, $m$-two-sided) hyperideal of $H$. This result likewise holds for distinct positive whole numbers.

Theorem 3.3. If $L$ is an $m$-left hyperideal of hypergroupoid $H$ and $R$ is an $m$-right hyperideal, $L \circ R$ is an $m$-hyperideal of $H$. 
Table 2 .

\begin{tabular}{ccc}
\hline$\circ$ & 1 & 2 \\
\hline 1 & $\{1\}$ & $\{1,2\}$ \\
2 & $\{1\}$ & $\{2\}$ \\
\hline
\end{tabular}

Proof. Because $H^{m} \circ L \circ R \subseteq L \circ R$, and $L \circ R \circ H^{m} \subseteq L \circ R$, $L \circ R$ is an $m$-hyperideal of $H$.

In the above case, product $R \circ L$ requires neither an $m$-left nor an $m$-right hyperideal of $H$.

Theorem 3.4. For some natural number $n$, the intersection of any collection of $m_{1}, m_{2}, \cdots$, and $m_{n}$-left (-right, -twosided) hyperideals of a hypergroupoid $H$ is a $t$-left (-right, -two-sided) hyperideal of $H$, where $t=\max \left(m_{1}, m_{2}, \ldots, m_{n}\right)$.

Proof. Clear.

Remark 3.8. For the intersection of an $m$-left hyperideal $L$ and an $m$-right hyperideal $R, L \cap R$ is not an $m$-hyperideal of $H$.

\section{Factorization of Hypergroupoids}

Hypergroupoids can be factorized with respect to their proper hyperideals, proper subhypergroupoids, and proper subsets. In this section, we mainly pursue factorization with respect to the subhypergroupoids and subsets. Thus, we define the factorizable, $(m, n)$-factorizable, generalized $(m, n)$-factorizable, $m$-factorizable, and generalized $m$-factorizable hypergroupoids in the context of their subhypergroupoids and subsets. In the following, we present the related concepts, examples, and necessary results.

Definition 4.1. A hypergroupoid $H$ is said to be factorizable if, for a proper subhypergroupoid $C$ of the hypergroupoid $H$, there exists another proper subhypergroupoid $D$ of $H$ having at least one distinct element from $C$, such that $C \circ D=H$. The pair $(C, D)$ is called the factorization of $H$ with factors $C$ and $D$ [12] and [13].

If the identity $e \in H$, pair $(e, H)$ is a factorization of $H$. The factorization of a hypergroupoid is not unique, as indicated by Example 4.2

Example 4.2. Consider the hypergroupoid $H=\{1,2\}$ with the hyperoperation $\circ$ defined in Table 2 The subhypergroupoids $\{1\}$ and $\{2\}$ form the factorization of $H$ as $\{1\} \circ\{2\}=\{1,2\}$. Moreover, $(\{1\},\{1,2\})$, and $(\{2\},\{1,2\})$ are also factors of $H$.
Proposition 4.3. If the right hyperideal $C$ and the left hyperideal $D$ form the factors of $H$, then $C \circ D=C \cap D$.

Proof. Because $C \circ D \subseteq C$ and $C \circ D \subseteq D, C \circ D \subseteq C \cap D$. However, if $C \cap D \subseteq C \circ D$ does not follow, then there exists $c \in C \cap D$ such that $c \notin C \circ D$. This gives $c \in H-C \circ D$, which is consistent with the fact that $(C, D)$ is a factorization of $H$, i.e., $C \circ D=H$.

Proposition 4.4. If a hypergroupoid $H$ contains either a strongly right magnifying or strong left magnifying element $a$, then $H$ is factorizable.

Proof. We prove this for the case of the strongly right magnifying element, the duality of which is analogously followed. We discuss two cases:

1. When $H$ is non-cyclic, suppose that $d$ is a strongly right magnifying element of $H$. In this case, $H=C \circ d$ for a proper subhypergroupoid $C$ of $H$. Because $H$ is not cyclic, it is factorizable because $H=C \circ D$, where $D=<d>$.

2. When $H$ is cyclic, let $H=<c>$. Letting $C$ be a proper subhypergroupoid of $H$, then $C=<c^{m}>$ for some positive integer $m$. Now, consider $H=C d=<$ $c^{m}>d$. Because $c$ is a generator of $H$, there exists a positive integer $p$ such that $d=c^{p}$. Thus, we have $H=<c^{m}>c^{p}=<c^{m+p}>=<c^{n}>$ for some integer $n=m+p$, which is a contradiction because $\left\langle c^{n}>\right.$ is a proper hypergroupoid of $H$. Thus, $H$ cannot be a cyclic hypergroupoid.

Relaxing the condition of the subhypergroupoid on the factors of a hypergroupoid leads us to define the generalized factors. This relaxion results in studying the non-empty subsets of hypergroupoids that form the factorization of the hypergroupoids.

Definition 4.5. A proper subset $L$ of the hypergroupoid $H$ is said to be a generalized factor of $H$ if there exists another proper subset $M$ of $H$ having at least one element distinct from $L$ such that $L \circ M=H$. The pair $(L, M)$ is called a generalized factorization of $H$ with the generalized factors $L$ and $M$, and the hypergroupoid $H$ is said to be a generalized factorizable hypergroupoid.

The following lemma is used in the proof of the preceding theorems characterizing factorizable hypergroupoids.

Lemma 4.6. Let $K$ be a set of all weak right-magnifying elements of a hypergroupoid $H$. If $K \neq \emptyset$, then $K$ is a 
subhypergroupoid of $H$. The complement of $K$ given by $J=H-K \neq \emptyset$ is also a subhypergroupoid of $H$ and $J \subseteq J \circ K$.

Proof. If $s, t \in K$, we can find two proper subsets $A$ and $B$ of $H$ such that $H=A \circ s=B \circ t$. This gives $A \circ s \circ t=$ $B \circ t \circ t=\circ t=H$ and $H=A \circ(s \circ t)$. Thus, $s \circ t \in K$ and $K$ is a subhypergroupoid. Let $u, v \in J$. If $u \circ v \in J$, the result immediately follows. If $u \circ v \notin J$, then $u \circ v \in K$, which contradicts the hypothesis that $u, v \notin K$. Hence, $u \circ v \notin J$ is invalid.

Letting $p \in J$ and $q \in K$, there is a proper subset $C$ in $H$ such that $H=C \circ q$. Moreover, there is an element $c$ in $C$, such that $p=c \circ q$. Now, if $c \in K$, then $p=c \circ q \in K$; this is a contradiction because $p \in K$. Thus, $c \in J$, and therefore $j \in J \circ K$ and $J \subseteq J \circ K$.

Theorem 4.1. Let $H$ be a hypergroupoid having a weak right magnifying element; thus, $H$ is generalized and factorizable if it satisfies any one of the following conditions:

1. $H$ has a left identity element,

2. $H$ has a weak left magnifying element,

3. $H$ is regular.

Proof. Letting $K=\{x: x$ is a weak right magnifying element of $H$ \}, it is obvious that $K$ is a subhypergroupoid of $H$. Let $J=H-K$

1. If $e$ is a left identity of $H$, because $e \notin K, J$ is a hypergroupoid and $J \subset J \circ K$ based on 4.6. In addition, $K=e \circ K \subseteq J \circ K$, and thus $H=J \cup K$ is generalized and factorizable as $H=J \circ K$.

2. Supposing that $y$ is a weak left magnifying element of $H$, we can then find two proper subsets $L$ and $M$ of $H$ such that $H=L \circ x=y \circ M$. Two cases need to be discussed.

(a) If $y \in K$, then $H=y \circ M=C \circ y$ for some proper subset $C$ of $H$, and $y=y \circ b=c \circ y$ for some $b \in M$ and $c \in C$. If $s$ is arbitrary in $H$, then $s=y \circ t=c \circ y \circ t=c \circ s$ for some $t \in M$. Thus, $c$ is a left identity of $H$. Thus, $H$ can be generalized as factorizable by part (1).

(b) Suppose that $y \notin K, J$ is then a hypergroupoid and $J \subseteq J \circ K$ based on 4.6 Furthermore, $L \subseteq J \circ K$. Assume $a \in L$; if $a \in J$, then $a \in J \circ K$. If
Table 3

\begin{tabular}{ccccc}
\hline$\circ$ & 1 & 2 & 3 & 4 \\
\hline 1 & $\{1\}$ & $\{2\}$ & $\{3\}$ & $\{4\}$ \\
2 & $\{2\}$ & $\{1,2\}$ & $\{4\}$ & $\{3,4\}$ \\
3 & $\{3\}$ & $\{4\}$ & $\{1,3\}$ & $\{3,4\}$ \\
4 & $\{4\}$ & $\{3,4\}$ & $\{2,4\}$ & $\{1,2,3,4\}$ \\
\hline
\end{tabular}

$a \in K$, then $a=y \circ d$ for some $d \in J \circ H$ However, $d \in K$, and thus $a \in J \circ K$. Hence, $H=L \circ x \subseteq(J \circ K) \circ K \subseteq J \circ K \subseteq H$, and thus $H$ is generalized factorizable as $H=J \circ K$.

3. If $H$ is regular, the set $E$ of its idempotents is then nonempty. Moreover, $E \cap K=\emptyset$; thus, $J$ is a hypergroupoid and $J \subseteq J \circ K$ by Lemma 4.6. Furthermore, if $y \in K$, then $y=\left(y \circ y^{\prime}\right) \circ y \in E \circ K \subseteq J \circ K$ for some $y^{\prime}$ in $H$, and $K \subseteq J \circ K$. Thus, $H=J \circ K$ is a generalized factorization of $H$.

Remark 4.7. Although the factors of a hypergroupoid are generalized, but the reverse does not hold.

Now, we present the ideas of $(m, n)$-factorizable and generalized $(m, n)$-factorizable hypergroupoids.

Definition 4.8. If there exist two proper subhypergroupoids $I$ and $J$ of the hypergroupoid $H$, having at least one distinct element, such that for any two positive integers $m$ and $n$, we have $I^{m} \circ J^{n}=H$, and $H$ is then said to be $(m, n)$-factorizable, and the pair $(I, J)$ is called an $(m, n)$-factorization of $H$ with the $m$-factors $I$ and $n$-factor $J$.

If $m=1$ and $n=1, H$ is said to be factorizable and generalized factorizable, as described in Definitions 4.1 and 4.5 respectively.

Definition 4.9. If there exist two proper subsets $L$ and $M$ of the hypergroupoid $H$ that have at least one distinct element, such that for any two positive integers $m$ and $n$, we have $L^{m} \circ M^{n}=H$, then $H$ is said to be generalized $(m, n)$ factorizable, and the pair $(L, M)$ is called the generalized $(m, n)$-factorization of $H$ with a generalized $m$-factor $L$ and generalized $n$-factor $M$.

Example 4.10. Consider the hypergroupoid $H=\{1,2,3,4\}$ with the hyperoperation $\circ$ defined in Table 3 The subsets $\{2\}$ and $\{3\}$ then form the $(2,2)$-factorization of $H$ as $\{2\}^{2} \circ$ $\{3\}^{2}=\{1,2\} \circ\{1,3\}=\{1,2,3,4\}$.

Example 4.11. In Section 5 , $\{A\}$ and $\{B\}$ are the generalized factors of $H=\{A, B, A B, O\}$ as $\{A\} \circ\{B\}=H$, and 
the set $H$ of $A B O$ is a generalized factorizable hypergroupoid whose only subhypergroupoid is $\{O\}$.

In Definitions 4.1 and 4.8 , without a loss of generality, by taking $m=n=1$, for a proper subhypergroupoid $I(L)$, the relation $I^{2}=I \circ I=H$ does not follow; however, this relation may hold for two proper subhypergroupoids (subsets) having at least one distinct element. This fact motivated us to present the idea of a generalized $m$-factorizable hypergroupoid for a positive integer $m$, as presented in the following:

Definition 4.12. A non-empty proper subset $K$ of $H$ is called a generalized $m$ factor of $H$, for a positive integer $m$, if $K^{m}=H$.

Definition 4.13. A hypergroupoid $H$ is said to be a generalized $m$-factorizable hypergroupoid if there exists an $m$-factor $L$ in $H$. In this case, $L^{m}=L \circ L \circ L \circ \cdots \circ \mathrm{E}$ is called the generalized $m$-factorization of $H$.

Because the concepts of maximality and minimality in the context of hyperideals play a vital role in characterizing a hypergroupoid, we define the concepts of the maximal and minimal generalized $(m, n)$-factors of hypergroupoids. These concepts will later be used to characterize hypergroupoids.

Definition 4.14. A generalized $m$-factor $M$ of $H$, for a positive integer $m$, is called a maximal generalized $m$-factor of $H$ if there exists no other generalized $m$-factor $N$ of $H$ such that $M \subseteq N$.

Definition 4.15. A generalized $m$-factor $M$ of $H$, for a positive integer $m$, is called a minimal generalized $m$-factor of $H$ if no other generalized $m$-factor $N$ of $H$ exists such that $N \subseteq M$.

Remark 4.16. Based on the pattern of Definitions 4.14 and 4.15, we can define the maximum and minimum factors for a hypergroupoid $H$.

Theorem 4.2. A hypergroupoid $H$ is simple if and only if $H$ is factorizable as $H=L \circ M$, where $L$ is the minimal left factor of $H$ and $M$ is the minimal right factor.

Proof. Suppose that $H$ is a simple hypergroupoid. Because $H \circ\{e\}$ and $\{e\} \circ H$ are the left hyperideal and right hyperideal, respectively, $H=(H \circ\{e\}) \circ(\{e\} \circ H), H$ is factorizable.

Conversely, suppose that $H=L \circ M$, where $L$ is the minimal left factor of $H$ and $M$ is the minimal right. We must show that $H$ is simple. For this, let $c=a \circ b \in H$. Because $L=H \circ a$ and $B=b \circ H, H=H \circ a \circ b \circ H=H \circ c \circ H$, which implies that $H$ is simple.

\section{ABO Blood Group the Key Genetics}

The blood groups of the offspring were determined based on their parents. There are almost 38 blood groups, but two are the most important with regard to blood transfusions. These were the $\mathrm{ABO}$ blood groups and $\mathrm{ABO} / \mathrm{Rh}$-factor blood groups. The percentages of blood groups in different racial groups worldwide are given in Table 4.

The most well-known and medically important blood types are the $\mathrm{ABO}$ blood types discovered by Karl Landsteiner in 1900 A.D. [14]. The ABO blood type of the offspring is controlled by a single gene, each of which is called the ABO gene with three types of alleles, namely $i, I_{A}$, and $I_{B}[15]$. The $I_{A}$ allele gives type $A, I_{B}$ gives type $B$, and $i$ gives type $O$. Because both $I_{A}$ and $I_{B}$ alleles are dominant over the $i$ allele, only $i i$ genotype people have type $O$ blood. Individuals with genotype $I_{A} I_{A}$ or $I_{A} i$ have type $A$ blood, individuals with genotype $I_{B} I_{B}$ or $I_{B} i$ have type $B$, and genotype $I_{A} I_{B}$ people have type $A B$. Parents with types $A$ and $B$ can also have a type $O$ offspring because their genotypes are $I_{B} i$ and $I_{A} i$, the combination of which can result in the $i i$ genotype.

Some red blood cells have an $\mathrm{Rh}$ factor, known as an $\mathrm{RhD}$ antigen. If the red blood cells contain the $\mathrm{RhD}$ antigen, they are $\mathrm{RhD}$ positive; otherwise, they are RhD-negative. Combining the effect of the $\mathrm{RhD}$ factor with the $\mathrm{ABO}$ blood group, an eight-blood-group-type system is obtained that consists of $A P$, $B P, A B P, O P, A N, B N, A B N$, and $O N$ blood types. Here, $P$ stands for positive, and $N$ stands for negative.

In this study, we considered the ABO blood groups and studied their genetics through hypergroupoids. The genetics of the $\mathrm{ABO} / \mathrm{Rh}$ blood group system can be studied using this pattern.

We keep the $\mathrm{ABO}$ blood groups in the set $H=\{A, B$, $A B, O\}$. Joining the hyperoperation 2.1 with set $H$ makes it a hypergroupoid. It should be noted that this hyperoperation is not associated with $H$. The hypergroupoid is expressed in Table 5

From Table 5, we extract the following information:

1. The samples $\{A, B\},\{A, B, O\},\{A, A B, O\},\{A, A B\}$, $\{B, A B\},\{A B, O\}$ and $\{B, A B, O\}$ are the generalized 2-factors of hypergroupoid $H$, e.g., $\{A, B\}^{2}=$ $\{A, B\} \circ\{A, B\}=\{A, B, A B, O\}$, as shown in Table 6 $\{A, B, A B\} \circ\{A, B, A B\}=\{A, B, A B, O\}$ shown in Table 7, and $\{A, B, O\}^{2}=\{A, B, O\} \circ\{A, B, O\}=$ $\{A, B, A B, O\}$, shown in Table 9 
Table 4. Percentage of blood groups around the world

\begin{tabular}{lcccccccc}
\hline Blood group & AP & BP & ABP & OP & AN & BN & ABN & ON \\
\hline African-American & 24 & 18 & 4 & 47 & 2 & 1 & 0.3 & 4 \\
Asian & 27 & 25 & 7 & 39 & 0.5 & 0.4 & 0.1 & 1 \\
Caucasian & 33 & 9 & 3 & 37 & 7 & 2 & 1 & 8 \\
Latino-American & 29 & 9 & 2 & 53 & 2 & 1 & 0.2 & 4 \\
\hline
\end{tabular}

Table 5. Blood groups depicted as hypergroupoid $H=\{A, B, A B, O\}$ under $\circ$

\begin{tabular}{ccccc}
\hline$\circ$ & $\mathrm{A}$ & $\mathrm{B}$ & $\mathrm{AB}$ & $\mathrm{O}$ \\
\hline $\mathrm{A}$ & $\{\mathrm{A}, \mathrm{O}\}$ & $\{\mathrm{A}, \mathrm{B}, \mathrm{AB}, \mathrm{O}\}$ & $\{\mathrm{A}, \mathrm{B}, \mathrm{AB}\}$ & $\{\mathrm{A}, \mathrm{O}\}$ \\
$\mathrm{B}$ & $\{\mathrm{A}, \mathrm{B}, \mathrm{AB}, \mathrm{O}\}$ & $\{\mathrm{B}, \mathrm{O}\}$ & $\{\mathrm{A}, \mathrm{B}, \mathrm{AB}\}$ & $\{\mathrm{B}, \mathrm{O}\}$ \\
$\mathrm{AB}$ & $\{\mathrm{A}, \mathrm{B}, \mathrm{AB}\}$ & $\{\mathrm{A}, \mathrm{B}, \mathrm{AB}\}$ & $\{\mathrm{A}, \mathrm{B}, \mathrm{AB}\}$ & $\{\mathrm{A}, \mathrm{B}\}$ \\
$\mathrm{O}$ & $\{\mathrm{A}, \mathrm{O}\}$ & $\{\mathrm{B}, \mathrm{O}\}$ & $\{\mathrm{A}, \mathrm{B}\}$ & $\{\mathrm{O}\}$ \\
\hline
\end{tabular}

2. The hypergroupoid $H$ is a 2-hypergroupoid because we can find two non-empty subsets of $H$ from which the hypergroupoid $H$ is obtained, as depicted in Tables 6 .

3. $\{A B, A B\}=\{A, B, A B\}$, meaning if both parents are $A B$ blood type, the offspring will be deficit of blood type $\mathrm{O}$, which is a universal donor.

4. Because $\{A, B, A B\} \circ\{O\}=\{A, B, A B, O\},\{A, B$, $A B\}$ and $\{O\}$ form the generalized factorization of $H$.

5. Because $\{A B\}^{2} \circ\{O\}=\{A, B, A B\} \circ\{O\}=\{A$, $B, A B, O\},\{A B\}$ and $\{O\}$ form a generalized $(2,1)$ factorization of $H$. This means that if one of the parents is blood type $A B$ and the other is blood type $O$, then their children will have a deficit of blood type $O$; however, their grandchildren will make the whole set $H$. Similarly, $\{A\}^{2} \circ\{A, B\}$ gives $\{A, B, A B, O\}$, implying that the children of $A$ and $A B$ will be deficient in blood type $O$; however, their grandchildren will make the whole blood groups.

6. $\{A\} \circ\{B\}=\{A, B, A B, O\}$, implying that $A$ and $B$ form the generalized factorization of $H$ and that $H$ can be composed by the coupled possessing of only $A$ and $B$ blood type groups.

7. The theory of the $m$-factorizable hypergroupoids (see Definition 4.13 seems more interesting, through which the effects of a single proper subset of the hypergroupoid can be seen in the composition of the hypergroupoid instead of two proper subsets. Because $\{A, B\}^{2}=$
Table 6.

\begin{tabular}{ccc}
\hline$\circ$ & $\mathrm{A}$ & $\mathrm{B}$ \\
\hline $\mathrm{A}$ & $\{\mathrm{A}, \mathrm{O}\}$ & $\{\mathrm{A}, \mathrm{B}, \mathrm{AB}, \mathrm{O}\}$ \\
$\mathrm{B}$ & $\{\mathrm{A}, \mathrm{B}, \mathrm{AB}, \mathrm{O}\}$ & $\{\mathrm{B}, \mathrm{O}\}$ \\
\hline
\end{tabular}

Table 7.

\begin{tabular}{cccc}
\hline$\circ$ & $\mathrm{A}$ & $\mathrm{B}$ & $\mathrm{AB}$ \\
\hline $\mathrm{A}$ & $\{\mathrm{A}, \mathrm{O}\}$ & $\{\mathrm{A}, \mathrm{B}, \mathrm{AB}, \mathrm{O}\}$ & $\{\mathrm{A}, \mathrm{B}, \mathrm{AB}\}$ \\
$\mathrm{B}$ & $\{\mathrm{A}, \mathrm{B}, \mathrm{AB}, \mathrm{O}\}$ & $\{\mathrm{B}, \mathrm{O}\}$ & $\{\mathrm{A}, \mathrm{B}, \mathrm{AB}\}$ \\
$\mathrm{AB}$ & $\{\mathrm{A}, \mathrm{B}, \mathrm{AB}\}$ & $\{\mathrm{A}, \mathrm{B}, \mathrm{AB}\}$ & $\{\mathrm{A}, \mathrm{B}, \mathrm{AB}\}$ \\
\hline
\end{tabular}

$\{A, B, A B, O\},\{A, B\}$ is a generalized 2-factor of $H$, the offspring of parents possessing blood types $A$ and $B$ make the complete blood group system.

8. Not all proper subsets or subhypergroupoids are factors, $(m, n)$-factors, generalized $(m, n)$-factors, or $m$-factors of $H$, including $\{A\}$ and $\{A B\}$, for example.

9. In hypergroupoid $H$, the $m$-factors $\{A, B\},\{A, A B\}$, and $\{B, A B\}$ are minimal generalized $m$-factors, whereas $\{A, B, A B\},\{A, B, O\},\{A, A B, O\}$ and $\{B, A B, O\}$ are maximal generalized $m$-factors.

10. The following hyperoperation $o_{c}$ determines the compatibility of the transfusion of the $\mathrm{ABO}$ blood groups.

$$
\begin{aligned}
& \circ_{c}=\{(A,\{A, A B\}),(B,\{B, A B\}), \\
& (A B,\{A B\}),(O,\{A, B, A B, O\})\} .
\end{aligned}
$$


Table 8. Blood groups of impossible children depicted as hypergroupoid $H=\{A, B, A B, O\}{\text { under }{ }_{i}}_{i}$

\begin{tabular}{ccccc}
\hline $\mathrm{o}_{i}$ & $\mathrm{~A}$ & $\mathrm{~B}$ & $\mathrm{AB}$ & $\mathrm{O}$ \\
\hline $\mathrm{A}$ & $\{\mathrm{B}, \mathrm{AB}\}$ & $\emptyset$ & $\{\mathrm{O}\}$ & $\{\mathrm{B}, \mathrm{AB}\}$ \\
$\mathrm{B}$ & $\emptyset$ & $\{\mathrm{A}, \mathrm{AB}\}$ & $\{\mathrm{O}\}$ & $\{\mathrm{A}, \mathrm{AB}\}$ \\
$\mathrm{AB}$ & $\{\mathrm{O}\}$ & $\{\mathrm{O}\}$ & $\{\mathrm{O}\}$ & $\{\mathrm{AB}, \mathrm{O}\}$ \\
$\mathrm{O}$ & $\{\mathrm{B}, \mathrm{AB}\}$ & $\{\mathrm{A}, \mathrm{AB}\}$ & $\{\mathrm{AB}, \mathrm{O}\}$ & $\{\mathrm{A}, \mathrm{B}, \mathrm{AB}\}$ \\
\hline
\end{tabular}

Table 9.

\begin{tabular}{cccc}
\hline$\circ$ & $\mathrm{A}$ & $\mathrm{B}$ & $\mathrm{O}$ \\
\hline $\mathrm{A}$ & $\{\mathrm{A}, \mathrm{O}\}$ & $\{\mathrm{A}, \mathrm{B}, \mathrm{AB}, \mathrm{O}\}$ & $\{\mathrm{A}, \mathrm{O}\}$ \\
$\mathrm{B}$ & $\{\mathrm{A}, \mathrm{B}, \mathrm{AB}, \mathrm{O}\}$ & $\{\mathrm{B}, \mathrm{O}\}$ & $\{\mathrm{B}, \mathrm{O}\}$ \\
$\mathrm{O}$ & $\{\mathrm{A}, \mathrm{O}\}$ & $\{\mathrm{B}, \mathrm{O}\}$ & $\{\mathrm{O}\}$ \\
\hline
\end{tabular}

The first ordered pair $(A,\{A, A B\})$ states that people with blood group $A$ can donate blood to people with blood groups $A$ and $A B$. Similarly, the other elements specify which blood group can be compatible with each other.

11. In cases of questioned paternity, the $A B O$ blood system is used to exclude a man from being a child's father. For example, a man with type AB blood cannot father a child with type $\mathrm{O}$ blood, as is evident in Table 8 , because he would pass on either the A or B allele to all of his offspring [16]. The $A B O$ blood groups cannot be used to confirm whether a man is indeed a child's father. Currently, DNA testing has superseded all other tests of paternity. However, it is used to exclude potential fathers rather than confirm the presence of a parental relationship.

Defining the hyperoperation $\circ_{i}: H \times H \rightarrow P^{*}(H)$ as $a \circ_{i} b=(a \circ b)^{c}$ for all $a, b \in H$, where $\circ$ is as defined in Table 5 we can see that $H=\{A, B, A B, O\}$ of the blood groups also creates a hypergroupoid under hyperoperation $\circ_{i}$, as shown in Table 8

12. The hypergroupoids $(H, \circ)$ and $\left(H, \circ_{i}\right)$ have different structures.

\section{6. $m$-Simple Hypergroupoids}

In the following, we characterize the $m$-right ( $m$-left) consistent simple hypergroupoids.
Definition 6.1. A hypergroupoid $H$ is called $m$-right ( $m$ left) simple if $H$ does not possess any proper $m$-right ( $m$-left) hyperideal contained in $H^{m}$. In other words, if $T$ is an $m$ right ( $m$-left) ideal of $H$ such that $T \subseteq H^{m}$, then $T=H^{m}$ [1], or equivalently, a hypergroupoid $H$ is called an $m$-simple hypergroupoid if it does not have any proper two-sided $m$ hyperideal, where a hyperideal, e.g., $A$, of a hypergroupoid $H$ is proper if $A \neq H$.

Example 6.2. The hypergroupoid $H=\{A, B, A B, O\}$ with the hyperoperation $\circ$ given in Table 5 is a simple hypergroupoid or 1-simple hypergroupoid because it does not possess any proper hyperideal.

Proposition 6.3. If $H$ is a hypergroupoid such that $a \circ H^{m}=$ $H^{m}\left(H^{m} \circ a=H^{m}\right)$ for every $a \in H$, then $H$ is $m$-right ( $m$-left) simple.

Proof. Let $a \circ H^{m}=H^{m}$ for every $a \in H$. To show that $H$ is $m$-right simple, let $T$ be an $m$-right ideal of $H$. In addition, let $b \in H$. Consider an element $t \in T(T \neq \emptyset)$. Because $t \in H^{m}$, by hypothesis, we have $t \circ H^{m}=H^{m}$. In addition, from $b \in H^{m}$, we have $b \in t \circ h$ for some $h \in H^{m}$. Therefore, we obtain $b \in t \circ h \subseteq T \circ H^{m} \subseteq T$, and thus $T=H^{m}$.

Similarly, if $H$ is $m$-left simple, then $H^{m} \circ a=H$ for every $a \in H$.

Remark 6.4. $H=\{A, B, A B, O\}$ follows Proposition 6.3

Definition 6.5. A hypergroupoid $H$ is said to be $m$-right consistent if, for every $a, b \in H,(a \circ b) \circ H^{m}=a \circ\left(b \circ H^{m}\right)$. Here, $H$ is called $m$-left consistent if, for every $a, b \in H^{m}$, $H^{m} \circ(a \circ b)=\left(H^{m} \circ a\right) \circ b$. If $H$ is both $m$-left consistent and $m$ right consistent, then it is called an $m$-consistent hypergroupoid.

Definition 6.6. A hypergroupoid $H$ is said to be $m$-intraconsistent if $\left(a \circ H^{m}\right) \circ b=a \circ\left(H^{m} \circ b\right)$ for all $a, b \in H$, for a positive integer $m$.

The following proposition describes the converse of Proposition 6.3

Proposition 6.7. Let $H$ be an $m$-right ( $m$-left) consistent hypergroupoid. If $H$ is $m$-right ( $m$-left) simple, then for every $a \in H$, we have $a \circ H^{m}=H^{m}\left(H^{m} \circ a=H^{m}\right)$.

Proof. Suppose $H$ is $m$-right simple and let $a \in H$. We must show that the set $a \circ H^{m}$ is an $m$-right hyperideal of $H$. For this, let $t \in\left(a \circ H^{m}\right) \circ H^{m}$. Thus, $t \in u \circ v$ for some $u \in a \circ H^{m}, v \in$ $H^{m}$. Because $u \in a \circ H^{m}$, we have $u \in a \circ w$ for some $w \in H^{m}$. Therefore, $t \in u \circ v \subseteq(a \circ w) \circ\{v\} \subseteq(a \circ w) \circ H^{m}$. However, $H$ is $m$-right consistent; therefore, $(a \circ w) \circ H^{m}=$ 
$\{a\} \circ\left(w \circ H^{m}\right)$. This implies that $t \in\{a\} \circ\left(H^{m} \circ H^{m}\right) \subseteq \circ H^{m}$. Therefore, $t \in \circ H^{m}$, and thus $\left(a \circ H^{m}\right) \circ H^{m} \subseteq \circ H^{m}$. This implies that $a \circ H^{m}$ is an $m$-right ideal of $H$. Because $H$ is $m$ right simple, we have $a \circ H^{m}=H^{m}$. If $H$ is $m$-left consistent, in a similar way, we then obtain $H^{m} \circ a=H^{m}$.

Remark 6.8. A right (left) $m$-consistent hypergroupoid $H$ is $m$-right ( $m$-left) simple if and only if, for any $a \in H^{m}$, we have $a \circ H^{m}=H^{m}\left(H^{m} \circ a=H^{m}\right)$.

Proposition 6.9. Let $H$ be an $m$-intra-consistent hypergroupoid. If $H$ is $m$-right ( $m$-left) simple, for every $a \in H$, we then have $a \circ H^{m}=H^{m}\left(H^{m} \circ a=H^{m}\right)$.

Proof. Let $H$ be $m$-right simple and $a \in H$. We need to show that the set $a \circ H^{m}$ is an $m$-right ideal of $H$. For this, let $t \in\left(a \circ H^{m}\right) \circ H^{m}$. Thus, $t \in u \circ v$ for some $u \in a \circ H^{m}$ and $u \in a \circ w$ for some $w \in H^{m}$. Then, we have that $t \in$ $u \circ v \subseteq(a \circ w) \circ\{v\}=(\{a\} \circ\{w\}) \circ\{v\} \subseteq\left(\{a\} \circ H^{m}\right) \circ\{v\}$. However, $H$ is $m$-intra-consistent, $\left(\{a\} \circ H^{m}\right) \circ\{v\}=\{a\} \circ$ $\left(H^{m} \circ\{v\}\right) \subseteq\{a\} \circ\left(H^{m} \circ H^{m}\right) \subseteq \circ H^{m}$. Thus, $t \in a \circ H^{m}$ and $a \circ H^{m}$ is an $m$-right ideal of $H$. Because $H$ is $m$-right simple, we have $a \circ H^{m}=H^{m}$. The dual case can be similarly proven.

On the patterns of Propositions 6.3 and 6.7, we have the following.

Proposition 6.10. An $m$-intra-consistent hypergroupoid $H$ is $m$-right ( $m$-left) simple if and only if, for any $a \in H$, we have $a \circ H^{m}=H^{m}\left(H^{m} \circ a=H^{m}\right)$.

Proof. Similar.

\section{Conclusion}

We presented the concepts of $m$-hyperideals, $m$-factors, and generalized $m$-factors in hypergroupoids. We also defined $m$ simple hypergroupoids and demonstrated these concepts by presenting examples from the mathematical fields as well as $\mathrm{ABO}$ blood groups. The genetics of the $\mathrm{ABO}$ blood type system were fully described based on the theory of hypergroupoids. A study on the eight blood groups can be further carried out based on the pattern found in studies on the ABO blood type.

The method of using hypergroupoids is easier and more applicable than other methods used in studies on the genetics of blood groups. In our research on the ABO example, the positive integers $m$ and $n$ refer to the successive generations, by which the effect of the blood groups of the parents on their children and grandchildren were discussed. However, we can take larger values of these two integers in a vast genetic example, similar to that of the gene effect on different generations. We used this idea to find samplings of blood groups, called $m$-factors, as well as generalized $m$-factors with reference to their maximality and minimality. This study can be extended to non-associative hyperstructures, such as those in [17,-19].

\section{Conflict of Interest}

No potential conflict of interest relevant to this article was reported.

\section{References}

[1] N. Kehayopulu, "How we pass from semigroups to hypersemigroups," Lobachevskii Journal of Mathematics, vol. 39, no. 1, pp. 121-128, 2018. https://doi.org/10.1134/ S199508021801016X

[2] F. Marty, "Sur une generalization de la notion de groupe," in Proceedings of 8th Congress of Scandinavian Mathematicians, 1934, pp. 45-49.

[3] A. Hasankhani, "Ideals in a semihypergroup and Green's relations," Ratio Mathematica, vol. 13, no. 1, pp. 29-36, 1999.

[4] N. Kehayopulu, "On fuzzy prime and fuzzy semiprime ideals of $\leq$-hypergroupoids," Journal of Hyperstructures, vol. 5, no. 2, pp. 108-114, 2016.

[5] S. Suebsung, T. Kaewnoi, and R. Chinram, "A note on almost hyperideals in semihypergroups," International Journal of Mathematics and Computer Science, vol. 15, pp. 127-133, 2020.

[6] M. Munir, N. Kausar, Salahuddin, and Tehreem, "On the prime fuzzy m-bi ideals in semigroups," Journal of Mathematics and Computer Science, vol. 21, no. 4, pp. 357-365, 2020. http://doi.org/10.22436/jmcs.021.04.08

[7] M. Munir, N. Kausar, B. Davvaz, M. Gulistan, and M. Gulzar, "Studying semigroups using the properties of their prime m-ideals," The Bulletin of Irkutsk State University. Series Mathematics, vol. 34, pp. 109-125, 2020. https: //doi.org/10.26516/1997-7670.2020.34.109

[8] M. Munir and A. Shafiq, "A generalization of bi ideals in semirings," Bulletin of the International Mathematical Virtual Institute, vol. 8, no. 1, pp. 123-133, 2018. 
[9] T. Kepka, "Ideals in selfdistributive groupoids," Commentationes Mathematicae Universitatis Carolinae, vol. 35, no. 1, pp. 187-191, 1994.

[10] D. Heidari, S. O. Dehkordi, and B. Davvaz, " $\Gamma-$ semihypergroups and their properties," University Politehnica of Bucharest Scientific Bulletin Series A, vol. 72, no. 1, pp. 195-208, 2010.

[11] N. Kehayopulu, "Green's relations for hypergroupoids," European Journal of Pure and Applied Mathematics, vol. 11, no. 3, pp. 598-611, 2018. https://doi.org/10.29020/ nybg.ejpam.v11i3.3306

[12] K. Tolo, "Factorizable semigroups," Pacific Journal of Mathematics, vol. 31, no. 2, pp. 523-535, 1969.

[13] D. Heidari and I. Cristea, "On factorizable semihypergroups," Mathematics, vol. 8, no. 7, article no. 1064, 2020. https://doi.org/10.3390/math8071064

[14] S. S. Kntha, "The blood revolution initiated by the famous footnote or Karl Landsteiner's 19001 paper," Ceylon Medical Journal, vol. 40, pp. 123-125, 1995.

[15] W. S. Klug, M. R. Cummings, and C. A. Spencer, Concepts of Genetics. Upper Saddle River, NJ: Prentice Hall, 2006.

[16] S. Nawaz, M. Gulistan, N. Kausar, and M. Munir, "On the left and right almost hyperideals of LA-semihypergroups," International Journal of Fuzzy Logic and Intelligent Systems, vol. 21, no. 1, pp. 86-92, 2021. https://doi.org/10. 5391/ijfis.2021.21.1.86

[17] N. Kausar, M. Alesemi, Salahuddin, and M. Munir, "Study on LA-ring by their intuitionistic fuzzy ideals," Mathematica Montisnigri, vol. 47, pp. 22-42, 2020.

[18] N. Yaqoob, M. Gulistan, J. Tang, and M. Azhar, "On generalized fuzzy hyperideals in ordered LA-semihypergroups," Computational and Applied Mathematics, vol. 38, no. 3, article no. 124, 2019. https://doi.org/10.1007/s40314-0190876-7

[19] M. Azhar, N. Yaqoob, M. Gulistan, and M. M. Khalaf, "On $\left(\epsilon, \epsilon \vee q_{k}\right)$-fuzzy hyperideals in ordered LAsemihypergroups," Discrete Dynamics in Nature and Society, vol. 2018, article no. 9494072, 2019. https://doi.org/ 10.1155/2018/9494072

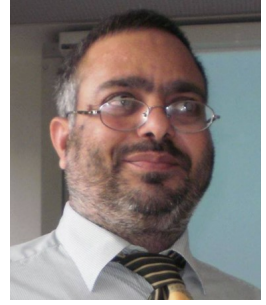

Mohammad Munir received his Ph.D. in applied mathematics from the University of Graz, Graz, Austria. His research interests lie in mathematical modeling of the biological system, fuzzy set theory, and its applications to multi-criteria decision making problems.

Nasreen Kausar received Ph.D. degree in Mathematics from Quaid-i-Azam university Islamabad, Pakistan. She is currently an Assistant Professor of Mathematics in Faculty of Arts and Science, Yildiz Technical University, Istanbul, Turkey. Her research interests are associative and commutative (non-associative and non-commutative algebraic structure and its applications on fuzzy structures.

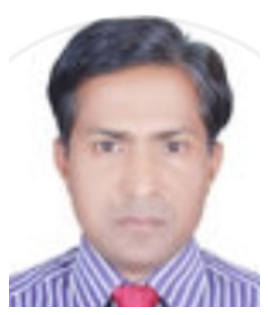

Salahuddin received a Ph.D. degree for his research in mathematics in 2001. He is working on fuzzy sets, fuzzy group theory, fuzzy ring and fuzzy ideal theory, variational inequalities, and optimization theory.

Rukhshanda Anjum obtained a Ph.D. degree in mathematics from Quaid-i-Azam University, Islamabad, Pakistan. Her research interests lie in the characterization of hemirings through the properties of their k-ideals.

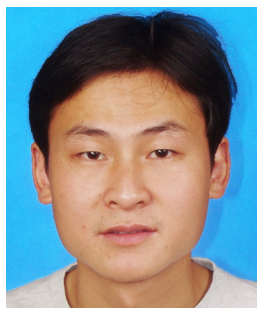

Qingbing Xu received his M.S. and degree from Fujian Normal University, Fuzhou, China, in 2008. His research interests include ring and module theory, homological algebra, category theory, semirings, and their applications.

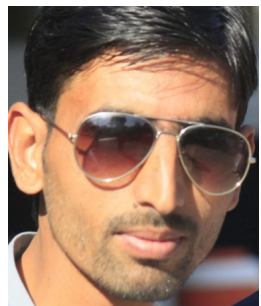

Waqas Ahmad is a lecturer in zoology in the Higher Education Department of Khyber-Pukhtunkhwa. He recently completed his M.Phil. His research interests lie in the fields of zoology, parasitology, and genetics. 